Effect of In doping in GaSb crystals studied by cathodoluminescence

This article has been downloaded from IOPscience. Please scroll down to see the full text article.

1999 Semicond. Sci. Technol. 14901

(http://iopscience.iop.org/0268-1242/14/10/304)

View the table of contents for this issue, or go to the journal homepage for more

Download details:

IP Address: 147.96.14.16

The article was downloaded on 21/02/2013 at 16:55

Please note that terms and conditions apply. 


\title{
Effect of In doping in GaSb crystals studied by cathodoluminescence
}

\author{
P Hidalgo $\dagger$, B Méndez $\dagger \|$, J Piqueras $\dagger$, P S Dutta $\ddagger$ and E Dieguez§ \\ $\dagger$ Departamento de Física de Materiales, Facultad de Físicas, Universidad Complutense, \\ E-28040 Madrid, Spain \\ \$ Department of Electrical, Computers and Systems Engineering, \\ Rensselaer Polytechnic Institute, Troy, NY 12180-3590, USA \\ $\S$ Departamento de Física de Materiales, Universidad Autónoma de Madrid, 28049 Madrid, \\ Spain
}

Received 6 May 1999, accepted for publication 5 July 1999

\begin{abstract}
The luminescence properties of In doped GaSb single crystals have been studied by the cathodoluminescence (CL) technique in the scanning electron microscope. It has been found that indium induces a certain reduction on the native acceptor concentration in contrast to what occurs with other isoelectronic dopants (e.g. aluminium). Large In concentrations lead to the formation of the ternary compound $\operatorname{In}_{x} \mathrm{Ga}_{1-x} \mathrm{Sb}$ as revealed by CL spectra and $\mathrm{x}$-ray measurements. In particular, a luminescence band and $\mathrm{x}$-ray diffraction peaks observed in highly doped samples are attributed to the presence of $\operatorname{In}_{x} \mathrm{Ga}_{1-x} \mathrm{Sb}$.
\end{abstract}

\section{Introduction}

The lattice-matched microstructures based in ternary and/or quaternary antimonide compounds have developed a great interest for the fabrication of long wavelength detectors and lasers. For this purpose high quality substrates are required and gallium antimonide appears as the most suitable material because of a small difference in lattice parameter in comparison with other III-V compounds. As-grown $\mathrm{GaSb}$ is always p-type in nature due to the native acceptors, which are complexes of gallium vacancies and gallium antisites $[1,2]$. One of the tasks of recent investigations in $\mathrm{GaSb}$ has been the reduction of native acceptor concentration by doping. However, significant progress is necessary to understand the influence of the different dopants on the native defects. In this work, In doped GaSb grown by the Bridgmann method has been characterized by the cathodoluminescence (CL) technique in the scanning electron microscope (SEM) which provides information on the nature and spatial distribution of the defects. This technique has been recently applied by our group to characterize aluminium doped GaSb crystals and the results show decoration of extended defects by Al but not a significant reduction of native acceptors [3]. In this work, a reduction of native defects beyond a threshold of indium concentration has been achieved. In samples with a larger concentration of In, a new luminescence band appears suggesting a different interaction between In and the defect structure in GaSb. The study has been completed with x-ray microanalysis and diffraction measurements to check the tentative suggestions about the $\mathrm{CL}$ results.

॥ To whom correspondence should be addressed.

\section{Experiment}

The samples studied in this work were GaSb:In crystals grown by the vertical Bridgmann method by tip nucleation without a seed (see references in [1]). The doping of the crystals was performed by adding In to the melt at the level of $10^{19} \mathrm{~cm}^{-3}$. The crystal used was $12 \mathrm{~mm}$ in diameter and about $40 \mathrm{~mm}$ in length. Four discs were cut perpendicular to the growth axis at various places along the length of the ingot and were chemo-mechanically polished to a mirror finish. The samples are labelled from 1 to 4 corresponding to positions from the bottom to the top of the crystal.

The CL measurements were carried out using a Hitachi S-2500 scanning electron microscope at $77 \mathrm{~K}$ and at accelerating voltages of $20-30 \mathrm{kV}$, by using a cooled $\mathrm{Ge}$ detector. The details of the experimental setup for spectral and panchromatic $\mathrm{CL}$ measurements are presented elsewhere [4]. In order to study the composition of some of the features observed in the SEM micrographs, x-ray diffraction measurements and mapping of the elements $\mathrm{Ga}, \mathrm{Sb}$ and In obtained by energy dispersive $\mathrm{x}$-ray microanalysis were performed in a JEOL JXA-8900 M superprobe.

\section{Results and discussion}

Our previous investigations show that the CL spectrum of undoped $\mathrm{GaSb}$ crystals exhibits two dominant transitions at 777 and $796 \mathrm{meV}$ [5]. The $777 \mathrm{meV}$ emission, called the A band, is usually attributed to the presence of acceptors [2] while the $796 \mathrm{meV}$ band corresponds to the band to band transition at $77 \mathrm{~K}$. Figure 1 shows the CL spectrum 


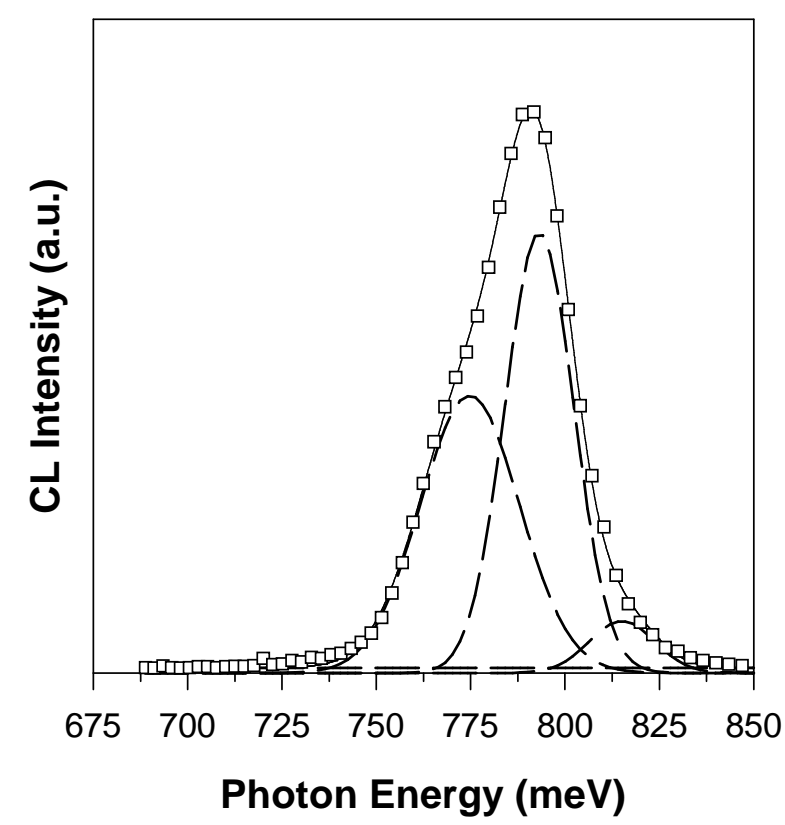

Figure 1. CL spectrum of a GaSb:In wafer grown by the vertical Bridgmann technique corresponding to sample 1. Symbols correspond to experimental data and broken lines to deconvoluted bands.

recorded in sample 1 , the bottom of the ingot, which presents the usual $\mathrm{GaSb}$ bands of undoped crystals, i.e. band gap transition $(796 \mathrm{meV})$, an intense defect band at $777 \mathrm{meV}$ and a weak above band gap emission $(815 \mathrm{meV})$ previously suggested [5] to be related to tail states and shallow acceptors. We have previously found a similar result in other doped GaSb Bridgmann crystals obtained from the bottom part of the ingots and in particular for Al doping [3,6]. Figure 2(a) shows the CL spectra obtained from the other three samples. A shift of the peak position is clearly detected from sample 2 (low In content) to sample 4 (higher In content). In each spectrum the different bands contributing to the observed peak have been determined from the best fits to the experimental data using a sum of Gaussian line distributions obtained by the Jandel Scientific Peakfit program (figures 2(b)-(d)). The result for sample 2 (figure 2(b)) shows an appreciable decrease of the acceptor band compared to sample 1 . In sample 3 (figure 2(c)) a new luminescence band appears at $763 \mathrm{meV}$ along with the defect band $(777 \mathrm{meV})$ and still a weak band gap transition (796 meV) luminescence is present. In sample 4 (figure 2(d)), the $763 \mathrm{meV}$ band dominates while the acceptor band has disappeared and there is a very weak contribution of the gallium antimonide band gap transition. Therefore there exits a threshold of indium concentration between samples 3 and 4 beyond which the native defects are strongly reduced and finally eliminated.

The CL images of In doped GaSb samples show an uniform bright background, similar to that found in undoped samples, and dark grain subboundaries. Depending on the sample considered, dark features may appear distributed in the bright background. In particular, the appearance of the CL images in sample 1 is similar to that of the undoped crystals [5]. In samples 2 and 3 a small density of small dark features is observed inside the grains while in sample 4 these precipitate-like features are uniformly distributed all over the sample. Figure 3 shows a representative CL image from sample 2 .

The different degree of incorporation of In, along the growth direction, is evidenced in the CL images and spectra obtained from the different samples. X-ray microanalysis of the regions with dark features in the different samples shows that the In content does not vary significantly. In fact, in samples 1 and 2 indium concentration is below the detection threshold and in samples 3 and 4 a small percentage of 1.4 and $1.8 \%$ respectively is found. These results indicate that the solubility of indium in gallium antimonide is rather high and the formation of precipitates has not taken place in contrast to what occurs with other elements in GaSb [6].

The presence of a new well resolved luminescence band in samples 3 and 4 , the disappearance of the peak related to band gap transition of $\mathrm{GaSb}$ and the good incorporation of In in the GaSb lattice without forming precipitates suggest that a ternary compound is formed at the top of the ingot during the growth process. In order to obtain more information on this possibility $\mathrm{x}$-ray diffraction measurements have been performed. In samples 1 and 2 the values of the lattice parameter obtained from the position of the angles of x-ray diffraction maxima correspond to the lattice parameter of GaSb (6.096 ̊). The most relevant finding has been that in samples 3 and 4 a shift in the position of diffraction maxima is detected and the lattice parameters measured from the $\mathrm{x}$ ray spectra were $6.101 \AA$ and $6.117 \AA$ respectively. This increase in the lattice parameter can be explained by the formation of a ternary compound $\operatorname{In}_{x} \mathrm{Ga}_{1-x} \mathrm{Sb}$. As is well known, even a small fraction of indium in the ternary leads to a decrease in the value of the band gap of the crystal. In figure 2 we have shown the evolution of the CL maxima found in our samples and the band at $763 \mathrm{meV}$ could correspond to the band gap of the ternary compound. The shift in the band gap due to the In incorporation in $\mathrm{GaSb}$ has been theoretically predicted to show a linear dependence on the fraction of In [7]. In our case, the band gap of $763 \mathrm{meV}$ would correspond to an In fraction of $x=0.02$, that is near the value of In percentage found in sample 4 by x-ray microanalysis. The appearance of dark features in samples 2 , 3 and 4 revealed by the CL images (figure 3 ) is related to the presence of indium but not to indium rich precipitates as concluded from $\mathrm{x}$-ray microanalysis. A possible origin of these features is the lattice mismatch between $\mathrm{InGaSb}$ and $\mathrm{GaSb}$ leading inside the grains. In fact, there is a problem in suppressing cracks in $\operatorname{In}_{x} \mathrm{Ga}_{1-x} \mathrm{Sb}$ crystals as recently reported [8]

Another interesting point is that isoelectronic doping of $\mathrm{GaSb}$ with In in an appropriate concentration is able to quench the luminescence band related to native defects. Previous investigations conclude that indium diffuses by a vacancy mechanism on the gallium sublattice [9]. According to those results the concentration of gallium vacancies and hence the native acceptor concentration would change with indium incorporation. In fact, we have found that the intensity of the $777 \mathrm{meV}$ band related to native defects decreases from samples 1 to 3 . In the case of sample 4, 


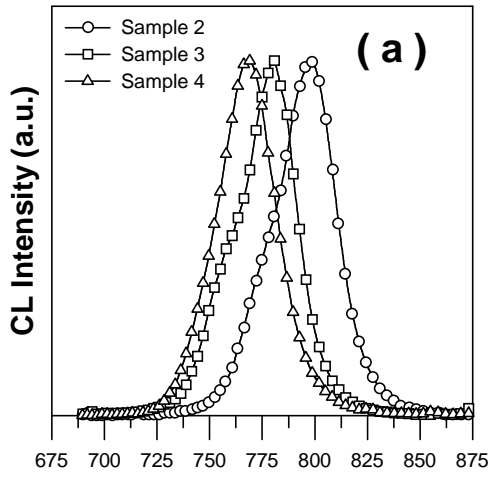

Photon Energy (meV)

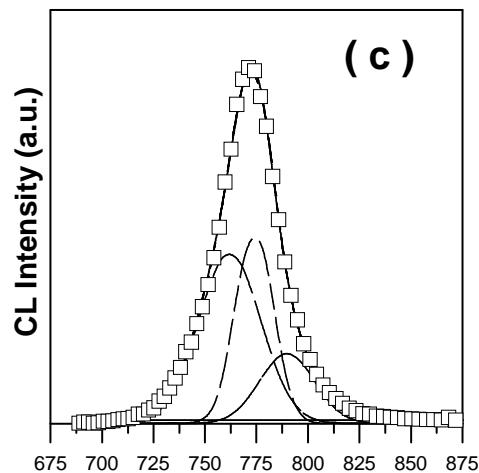

Photon Energy (meV)

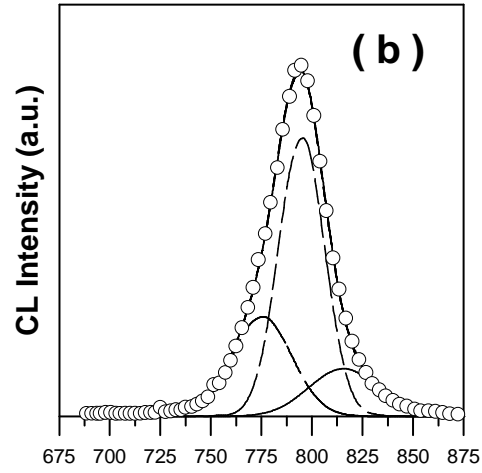

Photon Energy (meV)

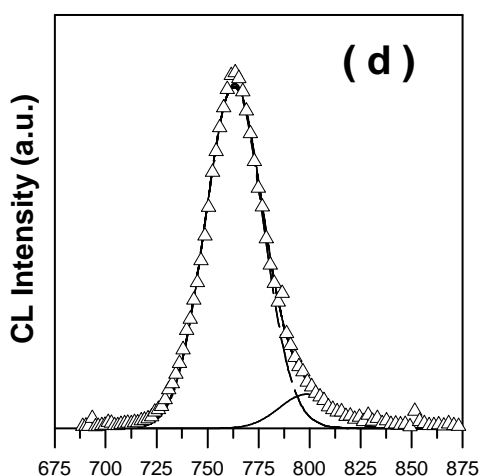

Photon Energy (meV)

Figure 2. (a) Evolution of CL spectra in GaSb:In along the growth direction, (b) CL spectrum of sample 2. A reduction of the acceptor band is detected, (c) CL spectrum of sample 3. The deconvolution evidences a new band at $763 \mathrm{meV}$ and (d) CL spectrum of sample 4 in which a dominant $763 \mathrm{meV}$ band is clearly present.

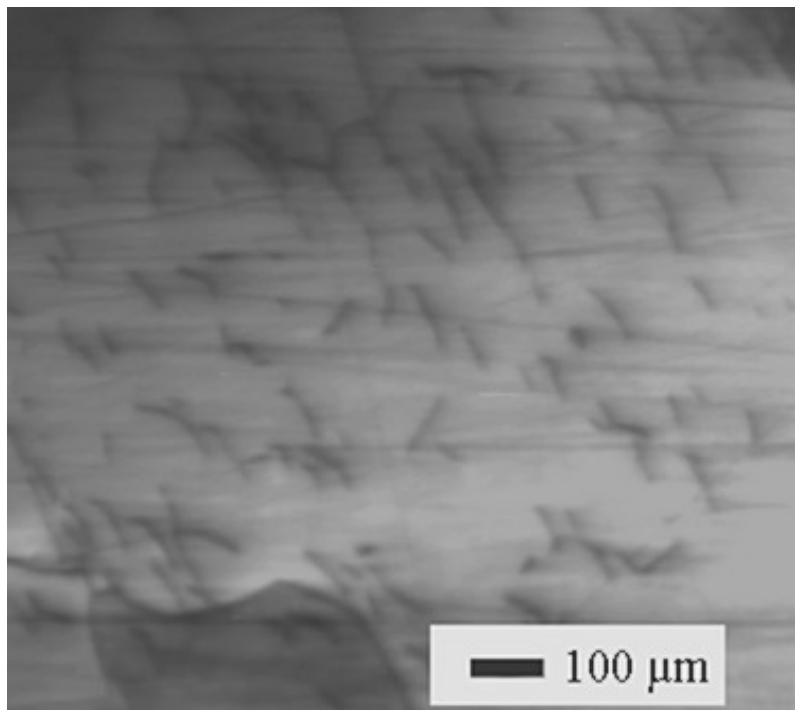

Figure 3. Representative CL image of sample 2.

the CL spectrum shows a single and intense band-gap related luminescence (figure 2(d)), indicating a good incorporation of In into the gallium sublattice leading to a ternary compound.

\section{Conclusions}

The efficiency of indium incorporation in the GaSb lattice was studied by CL spectroscopy and imaging, and by $\mathrm{x}$-ray microanalysis and diffraction. In contrast to other isoelectronic dopants, like $\mathrm{Al}$ in which native defects were not influenced, a reduction of these defects through the corresponding luminescence band has been observed. The effective suppression of native acceptors is achieved in the sample with higher indium content, beyond $1.4 \%$, in which $\mathrm{In}_{x} \mathrm{Ga}_{1-x} \mathrm{Sb}$ is formed. Future work with intermediate doping level (between samples 3 and 4) would result in a high quality $\mathrm{GaSb}$ with higher luminescence efficiency.

\section{Acknowledgments}

This work has been supported by the DGES (project No PB96-0639) and CICYT (projects ESP95-0148 and ESP98-1340).

\section{References}

[1] Dutta P S, Bhat H L and Kumar V 1997 J. Appl. Phys. 81 5821

[2] Lee M, Nicholas D J, Singer K E and Hamilton B 1986 J. Appl. Phys. $\mathbf{5 9} 2895$ 
P Hidalgo et al

[3] Hidalgo P, Méndez B, Piqueras J, Dutta P S and Dieguez E 1998 Solid State Commun. 108997

[4] Méndez B and Piqueras J 1991 J. Appl. Phys. 692776

[5] Méndez B, Piqueras J, Dutta P S and Dieguez E $1995 \mathrm{Appl}$. Phys. Lett. 672648
[6] Hidalgo P, Méndez B, Dutta P S, Piqueras J and Dieguez E 1998 Phys. Rev. B 576479

[7] Camassel J and Auvergne D 1975 Phys. Rev. B 12325

[8] Dutta P S and Ostrogorsky A G 1998 J. Cryst. Growth 1941

[9] Mathiot D and Edelin G 1980 Phil. Mag. A 41447 